\title{
Diseño y validación de un modelo para la evaluación de la calidad de la asistencia técnica agropecuaria en Colombia
} Design and validation of a model for evaluating the quality of
agricultural technical assistance in Colombia
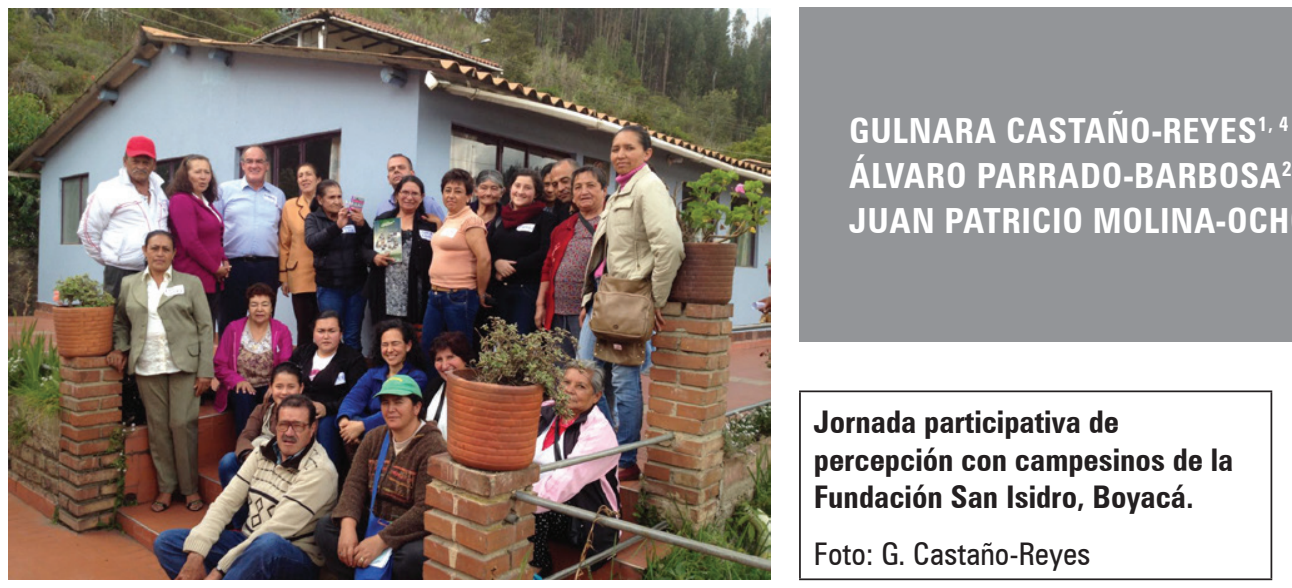

\section{RESUMEN}

El servicio de asistencia técnica agropecuaria en Colombia está reglamentado mediante leyes y decretos que definen los criterios para el seguimiento y verificación del desempeño de las entidades, sin embargo, se evidencian debilidades en la valoración de las variables que denotan calidad de la prestación del servicio. Debido a ello, fue diseñado el Modelo de Evaluación de la Calidad de la Asistencia Técnica Agropecuaria-MECATA, basado en la adaptación del modelo EFOM (European Foundation for Quality Management) y la identificación de variables mediante procesos participativos. Los resultados corresponden a la selección de 39 variables relacionadas con la calidad del servicio que conforman el modelo MECATA; perfeccionamiento de tres instrumentos de evaluación y un proceso de validación en dos entidades prestadoras del servicio en Boyacá. Se observa una valoración positiva de usuarios y asistentes técnicos sobre el servicio, sin embargo, la innovación de procesos, integralidad del servicio, formación y actualización, participación en otras actividades e integración al mercado obtuvieron deficiente calificación. El modelo MECATA es una herramienta que integra las perspectivas de los actores, identifica necesidades en el contexto y permite la formulación de propuestas de mejoramiento en la prestación del servicio de asistencia técnica cuando es requerido.

\begin{tabular}{r}
$\begin{array}{r}\text { Palabras clave adicionales: gestión participativa, legislación agropecuaria, calidad del } \\
\text { servicio, autoevaluación, mejoramiento continuo, método de análisis de contenido. }\end{array}$ \\
\cline { 2 - 2 }
\end{tabular}

Facultad de Ingeniería, Programa de Ingeniería Agroecológica, Corporación Universitaria Minuto de Dios (Uniminuto), Bogotá; Integrante Grupo de Investigación en Gestión y Desarrollo Rural (GIGDR), Universidad Nacional de Colombia, Bogotá (Colombia). ORCID Castaño-Reyes, G.: 0000-0002-2398-1265

2 Facultad de Medicina, Departamento de Nutrición Humana e integrante Grupo de Investigación en Gestión y Desarrollo Rural (GIGDR), Universidad Nacional de Colombia, Bogotá (Colombia). ORCID Parrado-Barbosa, A.: 00000002-7095-9693

3 Facultad de Ciencias Agrarias, Departamento de Desarrollo Rural e integrante Grupo de Investigación en Gestión y Desarrollo Rural (GIGDR), Universidad Nacional de Colombia, Bogotá (Colombia). ORCID Molina-Ochoa, J.P.: 00000002-9859-8148

4 Autor para correspondencia. gpcastanor@unal.edu.co 


\section{ABSTRACT}

Technical assistance programs for agriculture in Colombia are regulated by laws, agreements, and policies that define the importance of evaluating and monitoring this service. National, regional and municipal entities are responsible for developing performance evaluations; however, several weaknesses are evident. This paper proposes the Quality Assessment Model of Agricultural Advisory Services (known in Spanish as MECATA), an evaluation tool based on the adaptation of an EFQM model (European Foundation for Quality Management). The MECATA model was developed from the selection of 39 variables related to service quality; this model considered the adjustment of three assessment tools and a validation process of two entities in Boyacá. The results showed a positive service assessment given by users and technical assistants, but lower qualifications were considered in variables such as process innovation, integral service, continued education, participation in other activities and market inclusion. MECATA proposes a quality assessment that integrates several perspectives of technical assistance services based on local community requirements; these results can be used as the basis for proposals for improvement.

Additional key words: participative management, agricultural law, quality service, selfassessment, continuous improvement, qualitative content analysis.

Fecha de recepción: 10-06-2017 Aprobado para publicación: 30-09-2017

INTRODUCCIÓN

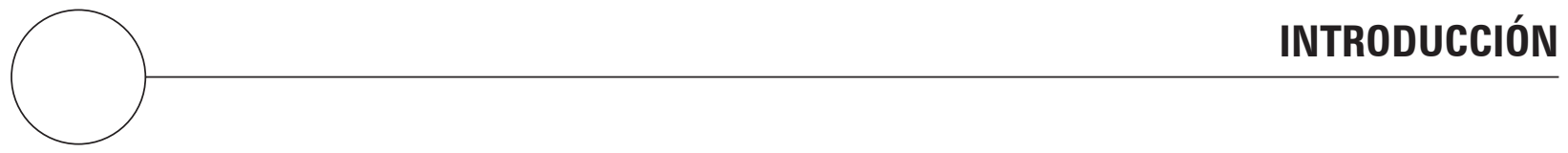

Los procesos de seguimiento y evaluación para programas de extensión y asistencia técnica agropecuaria en países latinoamericanos, poseen debilidades debido a que se realizan principalmente con base en los registros de procesos e indicadores de cumplimiento de proyectos (RELASER, 2012). Dichos programas son considerados un servicio público, donde los usuarios pueden evaluarlos en cuanto a su calidad (Cuevas et al., 2012).

La asistencia técnica agropecuaria (ATA) es considerada como un mecanismo que facilita la implementación o adopción de procesos técnicos y/o tecnológicos, por parte de los productores rurales y campesinos que garantiza su competitividad (Corpoica, 2015). La calidad en la prestación del servicio se reconoce como un principio básico de la ATA en la Ley 607/2000, siendo las secretarías de agricultura municipal y departamental, las responsables de realizar su seguimiento y evaluación (MADR, 2002). Este proceso debe realizarse en cada municipio acorde con los lineamientos del Plan General de Asistencia Técnica (PGAT), que incluye cobertura, calidad y pertinencia de la prestación del Servicio de Asistencia Técnica Agropecuaria (SATA).

En Colombia las secretarías de agricultura municipal evalúan el desempeño de las entidades prestadoras de SATA en el proceso de acreditación anual, basado en el cumplimento contractual y el PGAT municipal (MADR, 2005). Sin embargo, esta evaluación carece de elementos específicos que evalúen la calidad del servicio de manera participativa.

Existen dos evidencias de evaluación de la calidad del SATA. La primera evaluó las Umatas a nivel nacional (Vargas del Valle et al., 1996) a partir del análisis de variables como satisfacción, métodos de extensión e indicadores de cobertura. La segunda, corresponde a la experiencia del departamento de Antioquia, que desde el 2013, evalúa el desempeño de las entidades prestadoras del SATA en cumplimento de disposiciones legales, análisis de percepción de usuarios, pertinencia de los métodos de extensión, apropiación de nuevos conocimientos y bienestar de las comunidades rurales (Gobernación de Antioquia, 2014). Estas evaluaciones evidencian vacíos de información sobre fortalezas, dificultades y ausencia de planes de mejoramiento estratégico y continuo del SATA.

Como resultado de la identificación de las debilidades de la evaluación de la ATA, se propone el Modelo MECATA, que debido a su diseño contextualizado y participativo cumple el objetivo de ser una herramienta versátil para evaluar su calidad en entidades prestadoras a cualquier nivel. El modelo tiene la ventaja 
de plantear de manera descriptiva cada variable de percepción, mediante grados de cumplimento que permiten identificar la idoneidad del servicio de asistencia técnica y el cumplimento de la normatividad.

\section{MATERIALES Y MÉTODOS}

\section{Diseño metodológico}

El modelo EFOM aprovecha el intercambio de experiencias a través de la difusión de los parámetros fundamentales preestablecidos entre las organizaciones, con el objetivo de promover la excelencia. Está compuesto por nueve criterios: los primeros cinco corresponden a los "agentes facilitadores" que involucran todas las divisiones de la organización y permiten determinar las interacciones entre el liderazgo, gestión de las personas y de la estrategia. Los cuatro criterios restantes corresponden a los "resultados" que se dimensionan a partir de los usuarios, las personas y la sociedad, además de resultados clave que analizan planeación estratégica y cumplimiento de objetivos. Este modelo puede ser aplicado como método de cuestionario, de matriz de mejora, de reunión de trabajo, de formulario o de simulación de presentación al premio de excelencia (Cabo et al., 2014).

El modelo MECATA se desarrolla a través de 3 fases: (1) Identificación de variables directamente relacionadas con la calidad del SATA, (2) Diseño de los instrumentos de evaluación, (3) Validación y ajuste de instrumentos diseñados.

El diseño de la investigación es de tipo exploratorio-descriptivo no experimental, de corte transversal en grupos focales. Donde se indagaron las variables que influyen en la calidad del servicio de asistencia técnica. La recolección y análisis de información se realizó mediante Diagnóstico Rápido Participativo (DRP) (Lima de Almeida y Gaia, 2014) para la identificación de conceptos, opiniones y posibles soluciones por medio del autodiagnóstico.

\section{Identificación de variables relacionadas con la calidad del SATA}

La identificación de variables fue realizada a través de dos técnicas participativas aplicadas, la primera corresponde a una pregunta formulada en el Foro Nacional Linkata a nivel nacional, así: Desde su punto de vista y experiencia en Asistencia Técnica Agropecuaria,
¿Qué características debe poseer y qué elementos se deberían valorar para considerarla de buena calidad? Se realizó la transcripción de respuestas y con el método de análisis de contenido se extrajeron las frases con sentido, se desarrollaron matrices del análisis a profundidad e identificaron categorías emergentes (Miles et al., 2013; Jiménez et al., 2016).

La segunda técnica empleada fue, la Técnica Nominal de Grupo Participativa (TNGP) aplicada a nivel municipal, con priorización de los planteamientos individuales y ponderación para clasificación participativa y grupal (Álvarez, 1989). En este proceso, participaron promotores y asistentes técnicos de dos entidades prestadoras del servicio de asistencia técnica directa rural (Epsagro) del departamento de Boyacá.

\section{Instrumentos de evaluación del modelo MECATA}

En el modelo MECATA, el criterio "resultados en usuarios" se valora en dos etapas: la primera, describe la percepción de los usuarios del servicio brindado en los aspectos de confianza y aceptación del asistente técnico, pertinencia del servicio, atención de necesidades de manera oportuna, transmisión de la información de manera adecuada, flexibilidad, creatividad, integralidad del servicio y heterogeneidad; la segunda, corresponde al rendimiento de la continuidad del servicio, satisfacción del servicio y demanda del servicio.

Las etapas de evaluación del criterio "resultados en personas" corresponden a la percepción de los asistentes técnicos sobre la entidad. Se valora el trabajo en equipo, gestión organizativa, capacitación interna, apoyo a la capacitación y diálogo de saberes. El rendimiento evaluado corresponde a planeación estratégica, difusión del conocimiento, nivel de formación y actualización.

El criterio "resultados en la sociedad" es realizado en la autoevaluación de la entidad, en donde se evalúa la percepción mediante la imagen general, participación en actividades no agropecuarias, efecto sobre la producción, integración al mercado, conservación del medio ambiente y mejoramiento de la calidad de vida. El rendimiento evalúa las certificaciones recibidas por la entidad, contrataciones realizadas, apoyos externos recibidos, acuerdos y reconocimientos obtenidos.

El criterio "resultados clave" evalúa la gestión de la entidad sobre sus procesos internos: evaluación y seguimiento, cumplimiento del perfil organizacional y 
legislación nacional. El criterio de "rendimiento" valora los procesos asociativos, adopción de innovaciones, cobertura de usuarios y participación en mercados.

La valoración de cada criterio del modelo MECATA se desarrolla empleando las ecuaciones de 1 a 4, en las que cada peso específico de criterio (PCr) identificado en la tabla 1. La valoración final de la entidad obtenida con el modelo MECATA (Ec. 5) es la sumatoria de los resultados calculados para cada uno de los criterios.

$$
\begin{aligned}
& \begin{array}{c}
\text { Criterio resultados } \\
\text { en Usuarios }
\end{array}=\begin{array}{c}
P C r U \times(75 \% M P U \\
+25 \% I R U
\end{array} \\
& \begin{array}{c}
\text { Criterio resultados } \\
\text { en el Personal }
\end{array}=\begin{array}{c}
P C r P \times(75 \% M P P \\
+25 \% I R P
\end{array} \\
& \begin{array}{c}
\text { Criterio resultados } \\
\text { en la Sociedad }=\quad \begin{array}{c}
P C r S \\
+
\end{array} \quad+75 \% \text { IRS }
\end{array} \\
& \begin{array}{c}
\text { Criterio resultados } \\
\text { en Clave }
\end{array}=\begin{array}{c}
P C r C \times(50 \% R C \\
+50 \% \text { IC }
\end{array} \\
& \text { Valoración final }=\quad \begin{array}{c}
\sum \text { Criterios resultados } \\
\left(\begin{array}{c}
\text { usuarios, personal, } \\
\text { sociedad, clave) }
\end{array}\right.
\end{array}
\end{aligned}
$$

\section{Validación y ajuste de instrumentos de evaluación}

La validación del modelo MECATA se realizó en los municipios de Pauna y Duitama por medio de la evaluación de la prestación del SATA a pequeños y medianos productores agropecuarios. La Epsagro Agroéxito Sugamuxi (EAS) atiende al primer municipio en cultivos de clima cálido (Cacao, plátano y cítricos) y ganadería doble propósito. La Fundación San Isidro (FSI), acompaña a productores de agricultura familiar del segundo municipio en cultivos de clima frío como mora, aromáticas y ganadería de leche.

La calidad del SATA fue evaluada en Pauna por 10 campesinos ( $1,5 \%$ de los usuarios beneficiarios) y 8 Asistentes técnicos (72\%) de la EAS; en Duitama la evaluaron 13 campesinos (8\% de la población beneficiada) y 2 promotores rurales ( $40 \%$ del total) de la FSI.

El grado de confiabilidad de los instrumentos para evaluar la percepción fue calculada mediante el Coeficiente de Cronbach. El instrumento de percepción de usuarios fue de 0,75 y el de asistentes técnicos de 0,83 . Se considera fiable cuando el valor del coeficiente es cercano a 1 (Panayides, 2013).

\section{RESULTADOS Y DISCUSIÓN}

\section{Variables relacionadas con la calidad del SATA}

En la primera etapa, a partir del análisis de contenido, se identificaron 57 variables que posteriormente se agruparon en 9 categorías emergentes así: características de los asistentes técnicos, servicio brindado, metodologías empleadas, políticas de ATA, red de actores, necesidades de recursos económicos, características de la investigación, de las entidades y la gestión organizativa.

En la segunda etapa, empleando la TNGP se identificaron 35 variables relacionadas con la calidad del SATA que fueron agrupadas acorde con las características de los asistentes técnicos, características del servicio, metodologías empleadas y efectos en la sociedad.

El modelo MECATA se conformó por 39 variables (Tab. 1), de las cuales 21 fueron obtenidas al contrastar las resultantes de los procesos participativos. Los 18 restantes, se adicionaron con base las que enfatizan en el cumplimento de objetivos y metas de las entidades incluidas en los criterios resultados del modelo EFOM. Las variables se seleccionaron en cada etapa por semejanza, frecuencia, no redundancia, pertinencia y soporte conceptual (Castaño, 2016).

En trabajos desarrollados en ambientes participativos por Landini (2012) y Gutiérrez et al. (2013) se identificaron características que son requeridas en el perfil del asistente técnico agropecuario y extensionista rural. Algunas de estas concuerdan con las incluidas en el modelo MECATA y son mencionadas de manera directa o intrínseca en entrevistas semiestructuradas realizadas a pequeños productores, extensionistas, funcionarios y expertos en servicios de extensión rural. Entre estas, se encuentran la capacidad de comunicación, fortalecimiento de la confianza generada, la eficiencia de cumplimiento de labores, implementación de procesos de innovación con el uso de metodologías pertinentes, individuales y participativas para la difusión efectiva del conocimiento, valoración e integración de saberes, influencia en la producción, capacitación de los extensionistas para proporcionar un servicio integral, liderazgo, asociatividad, trabajo en equipo, evaluación y seguimiento. 


\section{Instrumentos de evaluación del modelo MECATA}

La calidad del SATA se evalúa en dos etapas con tres instrumentos que incluyen las variables identificadas en la primera fase. Se valora la percepción de los usuarios, asistentes técnicos y se realiza la autoevaluación de la entidad.

En la etapa de evaluación de la percepción se utilizaron dos cuestionarios diseñados en escala Likert, con una escala de cinco opciones de respuesta que va desde: no cumple [1] a cumple totalmente [5]. El primer cuestionario valora la percepción del servicio recibido con nueve preguntas sobre la calidad del SATA (Tab.1 columna a). El segundo cuestionario evalúa la percepción de los asistentes técnicos prestadores del SATA mediante cinco preguntas relacionadas con las variables de la tabla, columna c.

Un tercer cuestionario evalúa los cuatros criterios, constituido por 39 preguntas que incluyen la definición de cada variable y las opciones de selección según grado aceptación en escala Likert. Se requieren como insumo los resultados de las evaluaciones de percepción de la primera etapa para contestar las preguntas relacionadas con las columnas a y c, así como, las bases de datos de la prestación del servicio para contestar las preguntas relacionadas con las columnas $b, d$, fy h, de la tabla 1.

En procesos de desarrollo rural con enfoque territorial se han desarrollado metodologías para la evaluación y planeación de proyectos bajo esquemas participativos a partir de la identificación y priorización de las potencialidades del territorio con intervención de actores locales (Terrones, 2013). Se destaca que el modelo MECATA, por medio de la evaluación de la percepción de usuarios y asistentes técnicos permite la participación de los directos implicados en el SATA. Los análisis cualitativo y cuantitativo desarrollados reducen subjetividad al proceso de evaluativo con atributos contextualizados.

\section{Validación de instrumentos de evaluación}

\section{Evaluación de la prestación del SATA de la Epsagro Agroéxito Sugamuxi (EAS)}

\section{Percepción de usuarios}

Los resultados muestran que el $77,8 \%$ de variables tuvieron valores superiores al 70\% (Fig. 1), lo cual indica satisfacción de los usuarios. Las variables con mayor

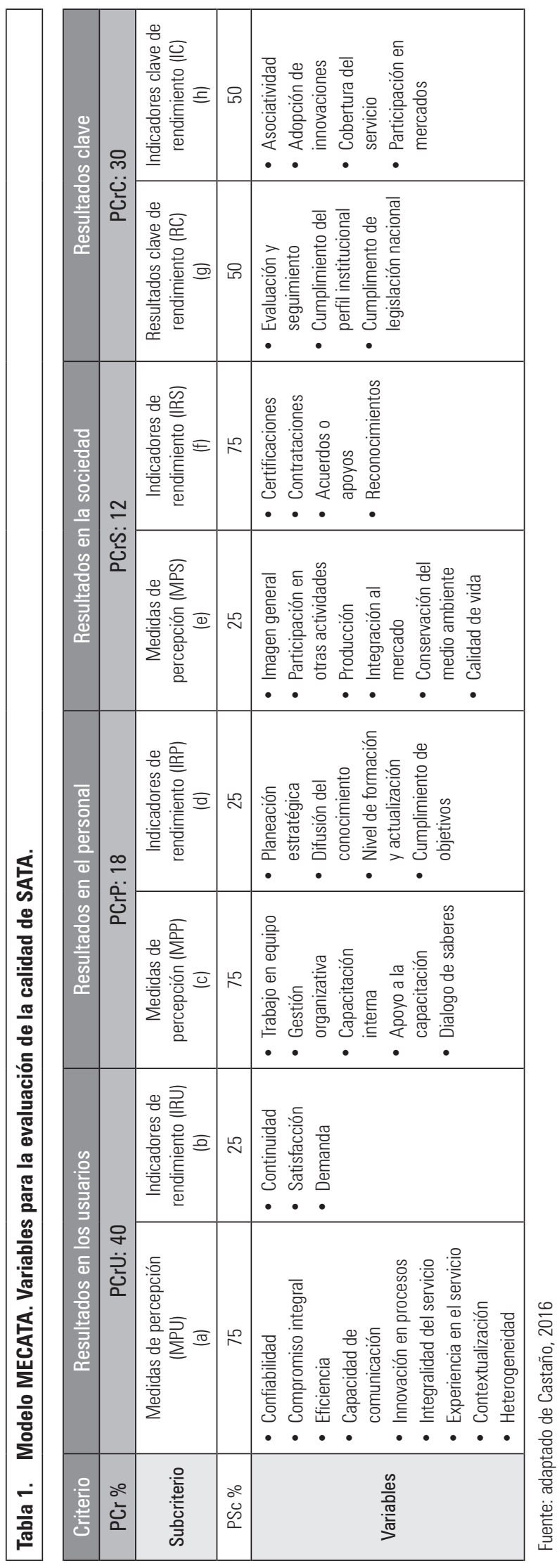

Vol. 11 - No. 2 - 2017 


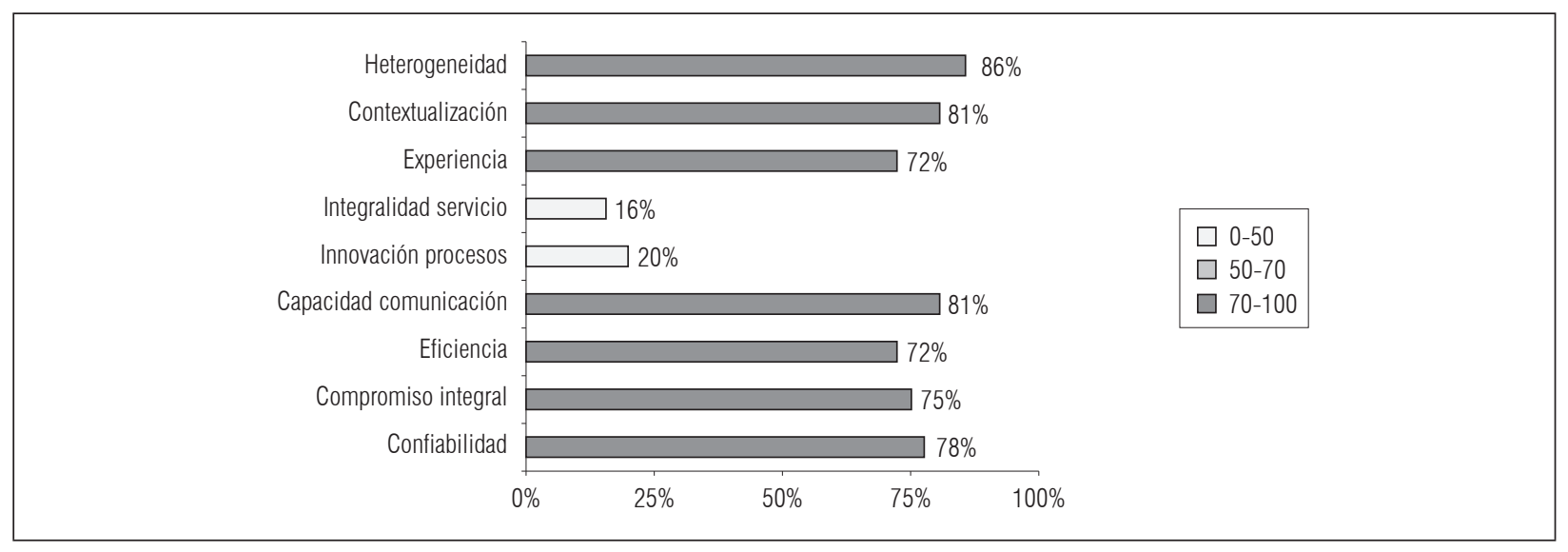

Figura 1. Percepción de la calidad del SATA. Usuarios EAS (adaptado de Castaño, 2016).

valoración fueron heterogeneidad, contextualización del servicio y capacidad de comunicación. En contraste, las variables innovación de procesos e integralidad del servicio obtuvieron puntajes bajos ( $\leq 20 \%)$. La valoración negativa de estas variables incluye la nula percepción de los componentes de creatividad y flexibilidad que conforman la variable innovación de procesos, así como los procesos de agregación de valor, aspectos económicos y políticos en la variable integralidad del servicio (Fig. 2).

\section{Percepción de asistentes técnicos}

Los resultados indican completa satisfacción en todas las variables evaluadas por los asistentes técnicos (Fig. 3 ) evidenciada por puntuaciones superiores al $80 \%$.

\section{Autoevaluación de la calidad del SATA}

El criterio "resultados en los usuarios" muestra que el $75 \%$ de las variables evaluadas poseen valores superiores al 70\%. La innovación en procesos por asistentes técnicos e integralidad del servicio requieren ser mejorados. En cuanto a los indicadores de rendimiento, se observa que la continuidad posee valores bajos lo cual se debe a la intermitencia en los procesos de contratación de la entidad e impacta en la calidad del servicio (Fig. 4A).

En el criterio "resultados en el personal" se evidencia una valoración alta para el 88,9\% de las variables evaluadas y, se observa completa satisfacción en la percepción de los asistentes técnicos. Para el indicador de rendimiento "nivel de formación y actualización" se muestra una deficiente calificación y debe ser tenido en cuenta en planes de mejoramiento por la entidad (Fig. 4B).

La valoración del criterio "resultados en la sociedad" indica que el $50 \%$ de las variables de percepción, muestran valores superiores al 70\% (Fig. 4C). Se infiere que las necesidades de la sociedad no están siendo consideradas a cabalidad por la entidad, razón por la cual se hace necesario mejorar acciones para la integración al mercado, participación en actividades no agropecuarias y procurar trabajar por influir en la calidad de vida de la comunidad a través del SATA.

El 57,1\% de las variables del criterio "resultados clave" obtuvieron valores inferiores al 70\%, lo cual indica que la entidad reconoce que existen aspectos que no abordan, los cuales se relacionan con el fortalecimiento de la entidad y cumplimento de normas internas. Así mismo, la asociatividad y la participación en mercados que hacen parte de actividades que deben ser cubiertas por el SATA según la legislación (Fig. 4D).

\section{Valoración final de la calidad del SATA}

La valoración final obtenida por EAS de 70,24\%, así: el criterio "resultados en el personal" obtuvo la mayor puntuación (88,3\%), lo cual indica que los asistentes técnicos están satisfechos con el apoyo que reciben por parte de la entidad y que se cumple con la mayoría de aspectos evaluados. El criterio "resultados en la sociedad" obtuvo $71,7 \%$ con altos valores en indicadores de rendimiento, aunque los aspectos identificados en percepción requieren ser mejorados. 


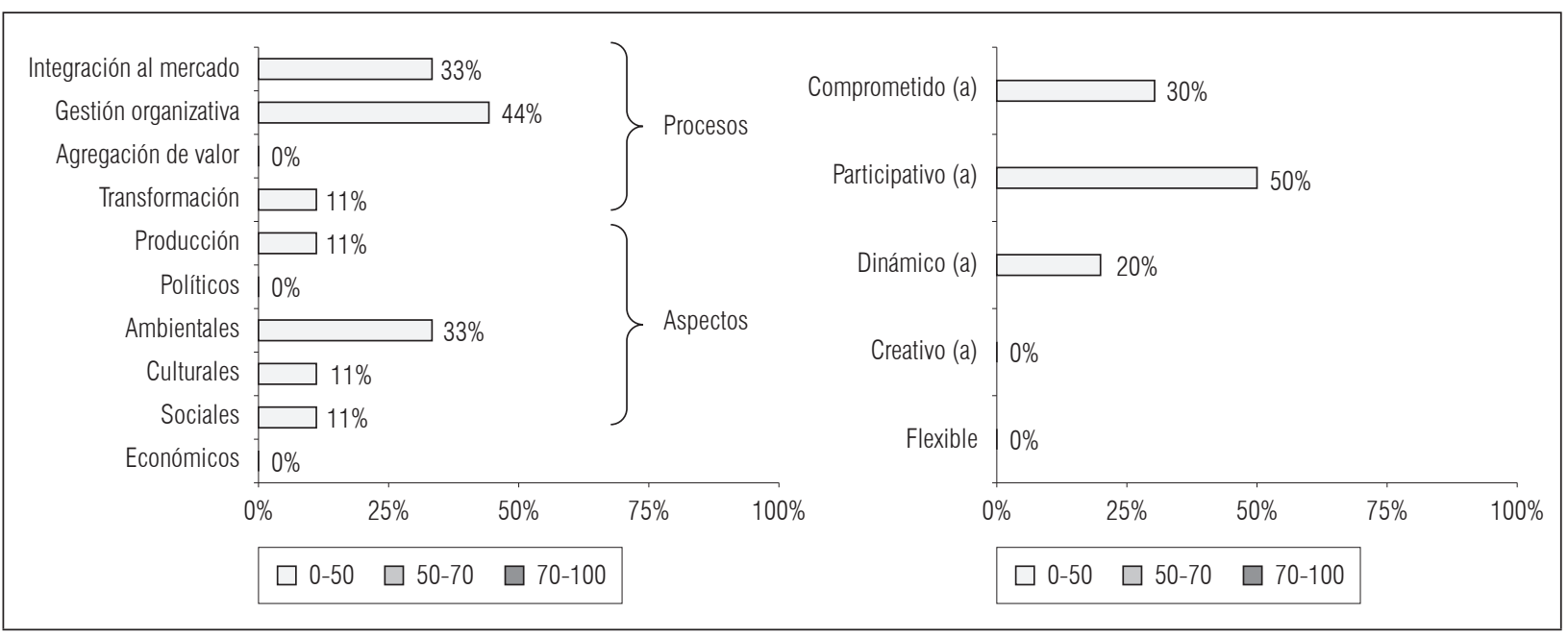

Figura 2. Percepción de Innovación e Integralidad. Usuarios EAS (adaptado de Castaño, 2016).

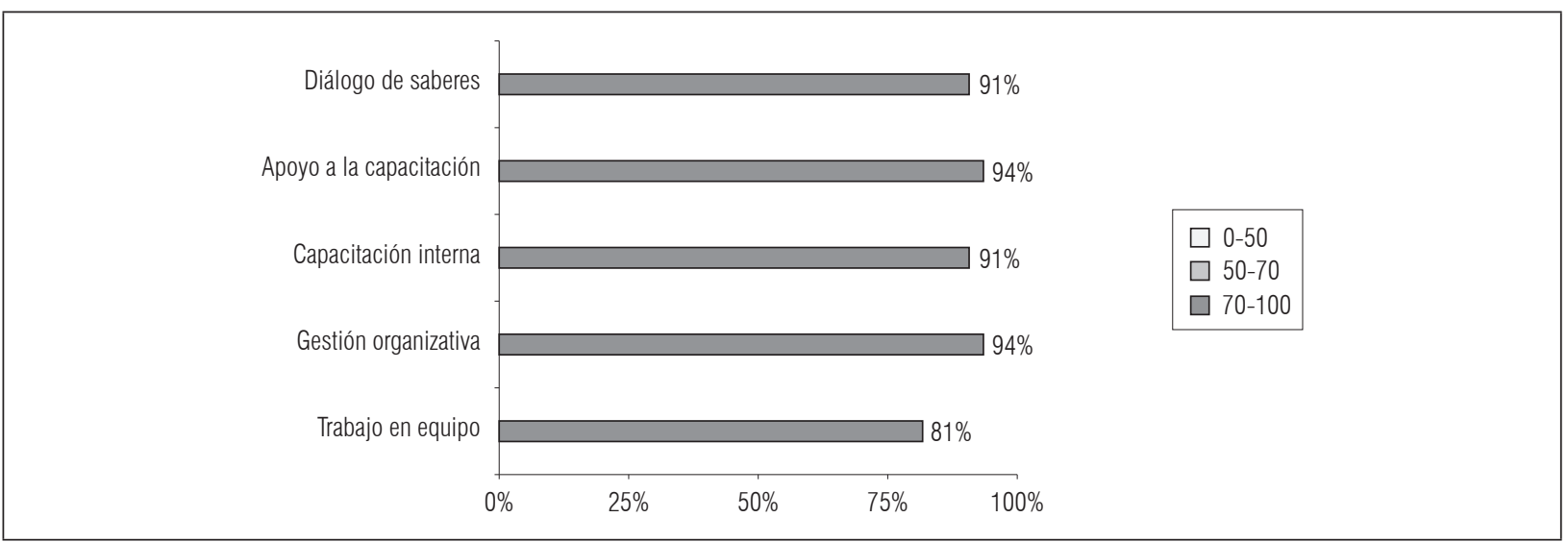

Figura 3. Percepción de la calidad del SATA. Asistentes técnicos EAS (adaptado de Castaño, 2016).

Los criterios "resultados en los usuarios" y "resultados clave" obtuvieron 68,7 y $60,8 \%$, respectivamente. En usuarios se presentan bajos valores en percepción y en los "resultados clave" deficiencias que igualmente requieren abordaje.

Finalmente, de acuerdo con los resultados arrojados por la autoevaluación, la EAS cumple con los objetivos de manera satisfactoria y posee un equipo de asistentes técnicos que brindan un servicio de buena calidad. Por otro lado, existen debilidades en las variables detectadas en cada criterio evaluado, razón por la cual la entidad planteó propuestas de mejoramiento prioritarias, entre las que se destacan: actualización al personal en métodos de extensión rural, temáticas integrales y específicas de cada profesión, identificar el entorno para diseñar estrategias eficientes, fomentar el liderazgo, asociatividad y mercados campesinos, entre otras.

\section{Evaluación de la prestación del SATA de la Fundación San Isidro, FSI}

\section{Percepción de usuarios}

El $66,7 \%$ de las variables analizadas fueron valoradas con puntajes superiores al 70\% (Fig. 5). Los valores superiores corresponden a heterogeneidad y contextualización del servicio, mientras que "integralidad del servicio" e "innovación de procesos" obtuvieron valores inferiores al 50\%. La baja calificación de estas variables es determinada por la baja percepción en los componentes creatividad y flexibilidad de la 


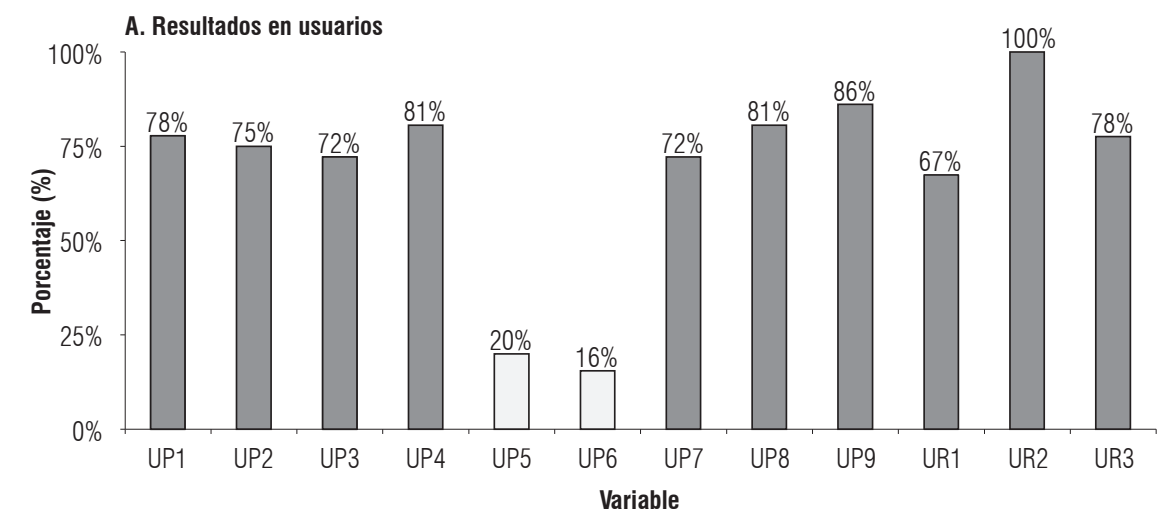

Resultados en usuarios

Medidas de percepción

UP1 Confiabilidad

UP2 Compromiso integral

UP3 Eficiencia

UP4 Capacidad comunicación

UP5 Innovación procesos

UP6 Integralidad servicio

UP7 Experiencia

UP8 Contextualización

UP9 Heterogeneidad

Indicadores de rendimiento

UR1 Continuidad

UR2 Satisfacción

UR3 Demanda

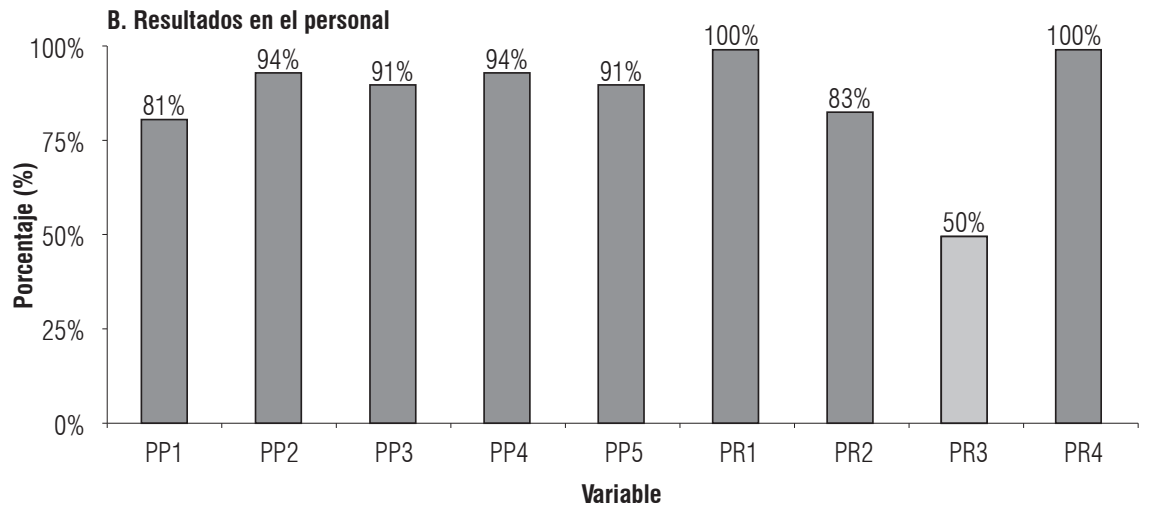

\begin{tabular}{|l|l|}
\hline \multicolumn{2}{|c|}{ Resultados en el personal } \\
\hline \multicolumn{2}{|c|}{ Medidas de percepción } \\
\hline PP1 & Trabajo en equipo \\
PP2 & Gestión organizativa \\
PP3 & Capacitación interna \\
PP4 & Apoyo a la capacitación \\
PP5 & Diálogo de saberes \\
\hline Indicadores de rendimiento \\
\hline PR1 & Planeación estratégica \\
PR2 & Difusión del conocimiento \\
PR3 & Nivel de formación y \\
& actualización \\
PR4 & Cumplimiento de \\
& objetivos \\
\hline
\end{tabular}

C. Resultados en la sociedad

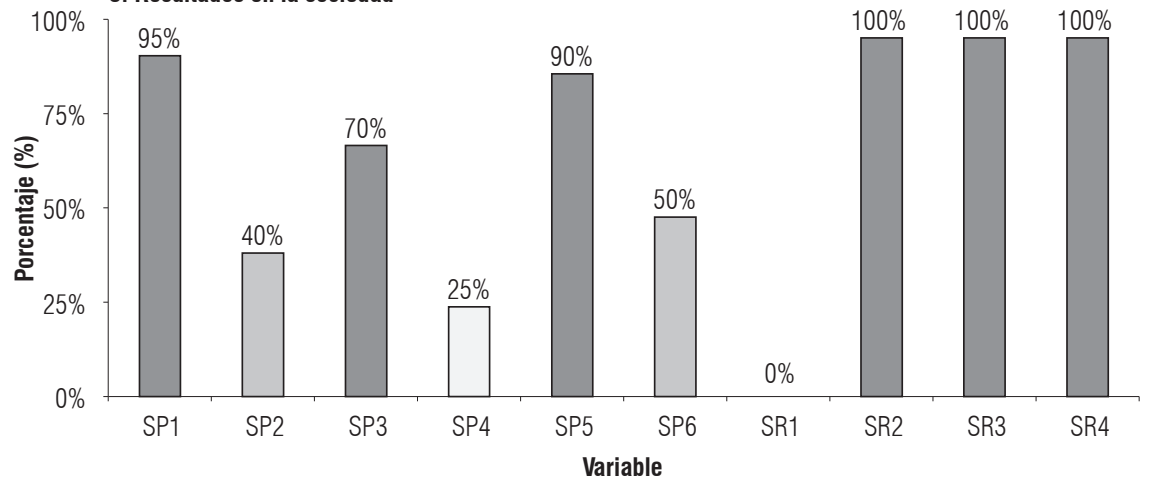

Resultados en la sociedad Medidas de percepción

SP1 Imagen general

SP2 Participación otras actividades

SP3 Producción

SP4 Integración al mercado

SP5 Conservación ambienta

SP6 Calidad de vida

Indicadores de rendimiento

SR1 Certificaciones

SR2 Contrataciones

SR3 Acuerdos 0 apoyos

SR4 Reconocimientos

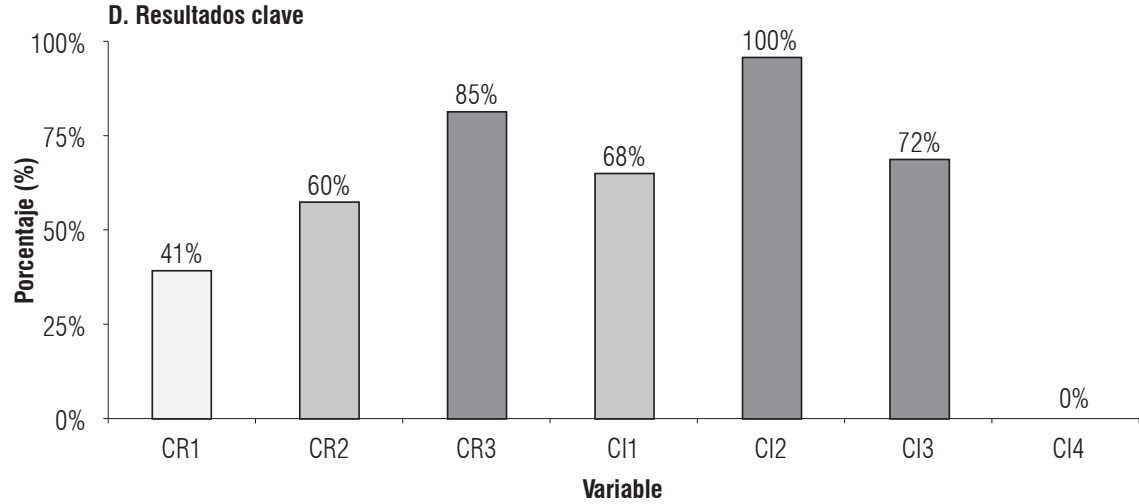

\begin{tabular}{|c|c|}
\hline \multicolumn{2}{|r|}{ Resultados clave } \\
\hline \multicolumn{2}{|r|}{ Resultados } \\
\hline CR1 & Evaluación y seguimiento \\
\hline CR2 & $\begin{array}{l}\text { Cumplimiento perfil } \\
\text { organizacional }\end{array}$ \\
\hline CR3 & $\begin{array}{l}\text { Cumplimiento legislación } \\
\text { nacional }\end{array}$ \\
\hline \multicolumn{2}{|r|}{ Indicadores } \\
\hline $\begin{array}{l}\mathrm{Cl} 1 \\
\mathrm{Cl} 2\end{array}$ & $\begin{array}{l}\text { Asociatividad } \\
\text { Adopción de } \\
\text { innovaciones }\end{array}$ \\
\hline $\mathrm{Cl} 3$ & $\begin{array}{l}\text { Cobertura } \\
\text { Participación en } \\
\text { mercados }\end{array}$ \\
\hline
\end{tabular}

Figura 4. Resultados de evaluación de la calidad de SATA. EAS. 
variable "innovación de procesos", así como, procesos de agregación de valor y transformación, además de los aspectos económicos y políticos en la variable "integralidad del servicio" (Fig. 6).

\section{Percepción por asistentes técnicos}

La percepción de los promotores de la FSI sobre la calidad del acompañamiento brindado por la entidad (Fig. 7) evidencia alta satisfacción en la variable

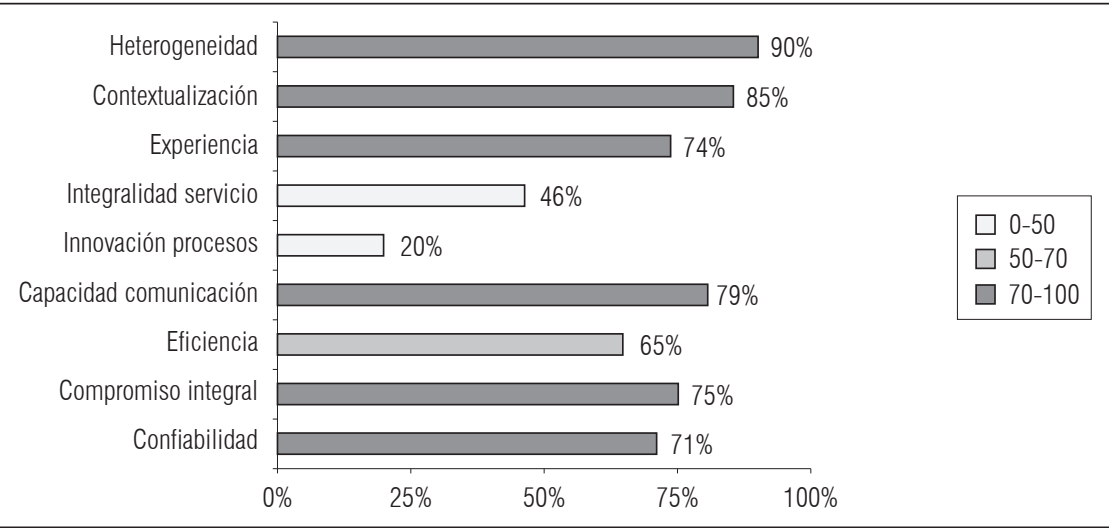

Figura 5. Percepción de la calidad del SATA. Usuarios FSI (adaptado de Castaño, 2016).

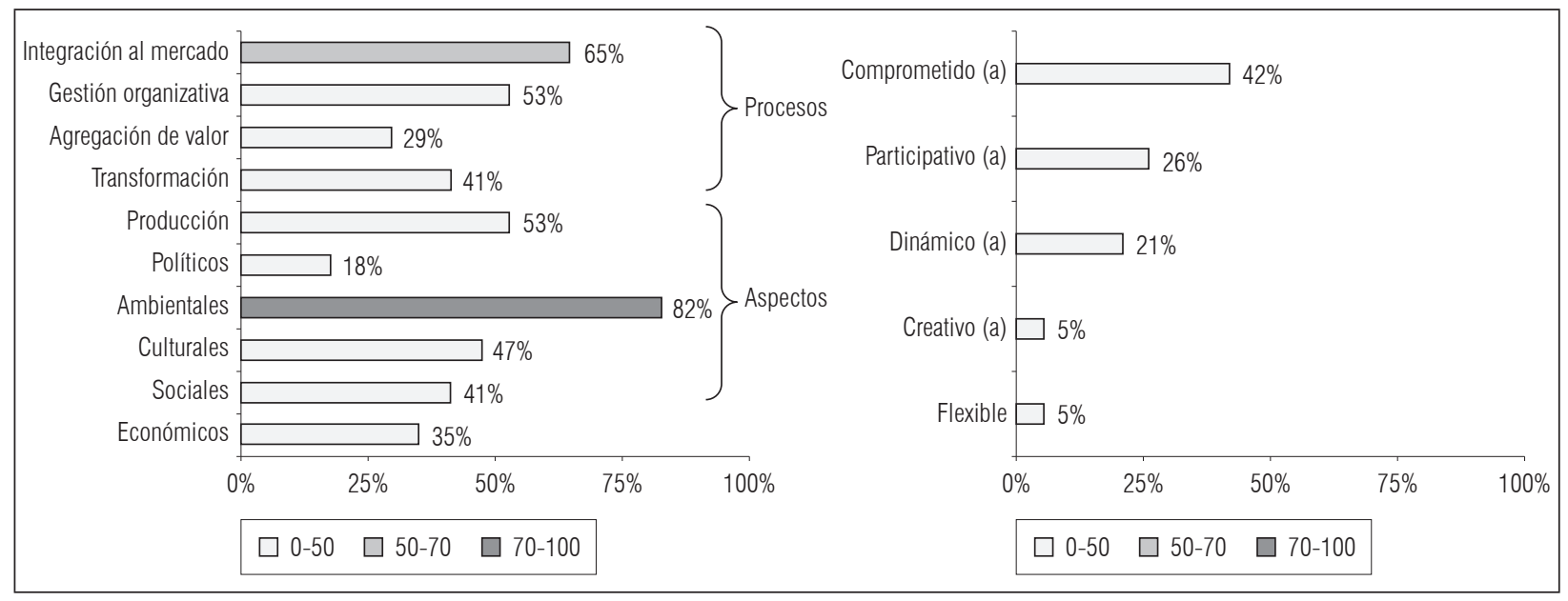

Figura 6. Percepción de Innovación e Integralidad. Usuarios EAS (adaptado de Castaño, 2016).

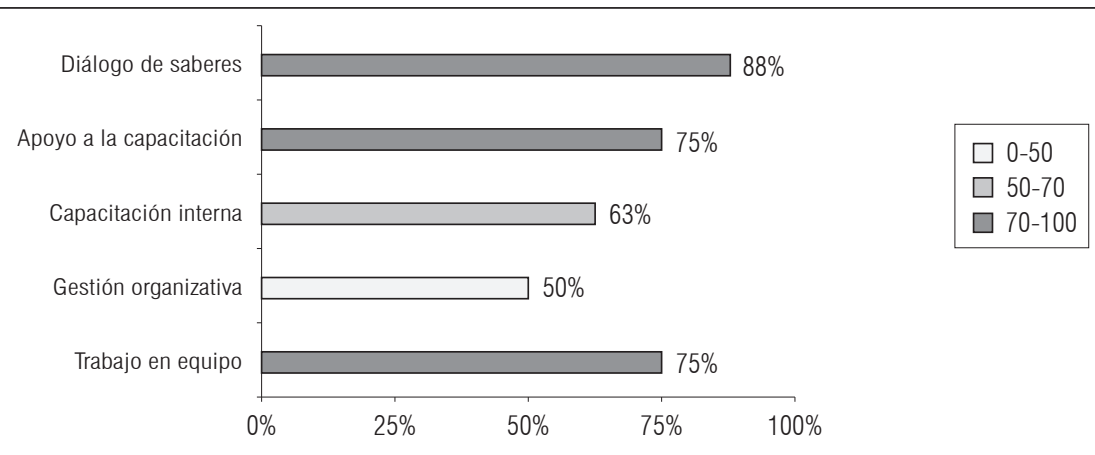

Figura 7. Percepción de la calidad del SATA. Asistentes técnicos FSI (adaptado de Castaño, 2016). 
"diálogo de saberes", mientras que en las variables "gestión organizativa" y "capacitación interna" se observan valores inferiores al $70 \%$.

En el estudio de Méndez (2015) se encontraron resultados equiparables con insatisfacciones de los asistentes técnicos en la variable "posibilidades de aprendizaje continuo" en las entidades en la cuales laboran.

\section{Autoevaluación de la calidad del SATA}

En el criterio "resultados en los usuarios" el 75\% de las variables evaluadas poseen valores superiores al $70 \%$. Esto indica que la entidad requiere implementar mejoras en la innovación en procesos por parte de los asistentes técnicos e integralidad del servicio. Por otro lado, los indicadores de rendimiento presentan alta valoración debido a continuidad del servicio brindado (Fig. 8A).

El criterio "resultados en el personal" posee valoración alta para el 55,6\% de las variables evaluadas. Percepción de "Gestión organizativa", "capacitación interna", rendimiento en "difusión del conocimiento" y "nivel de formación y actualización", obtuvieron bajas calificaciones (Fig. 8B). Se deduce que estos valores se obtuvieron porque los promotores son miembros asociados a la FSI y desean su mejoramiento para beneficio mutuo.

El criterio "resultados en la sociedad" muestra que el $80 \%$ de las variables tienen valores superiores al $70 \%$, lo que demuestra alto compromiso social de la entidad (Fig. 8C).

Las variables con alta valoración del criterio "resultados clave" corresponden a $71,4 \%$, lo que indica que la entidad cumple con metas, objetivos y normatividad establecidos. La variable "participación en mercados" $(41,2 \%)$ requiere fortalecerse (Fig. $8 D)$.

\section{Valoración final de la calidad del SATA}

La valoración final fue de $79,24 \%$, se identificó que el criterio "resultados en la sociedad" obtuvo mayor puntuación (94,8\%), lo cual refleja la misión social de la FSI. Los "resultados clave" obtuvieron $82,3 \%$ en valoración debido que cumplen las políticas y normatividad interna. Por otro parte, los "resultados en usuarios" (75,4\%) y "resultados en personal" (72,3\%) son el efecto de la labor comprometida de la entidad con sus miembros.
La autoevaluación permitió identificar que la FSI es una entidad cumplidora de su perfil organizacional, comprometida con la sociedad y que sus miembros tienen sentido de pertenencia y arraigo. Así mismo, la existencia de debilidades que fueron base para realizar propuestas de mejoramiento, como: formar el equipo en temáticas integrales; actualizar el equipo de trabajo en agroecología; elaboración de herramientas de seguimiento; evaluación y planeación del trabajo en equipo; actualización del equipo en metodologías participativas,; y gestión de proyectos para fortalecer la participación en mercados; entre otras.

Los resultados obtenidos en la validación para las dos entidades prestadoras de asistencia técnica agropecuaria evidencian que, tanto los usuarios como los asistentes técnicos, valoran positivamente la calidad de los servicios prestados, aunque se observan deficiencias marcadas en aspectos como innovación de procesos, integralidad del servicio, formación y actualización, participación en otras actividades y en mercados.

Se determina que la diferencia en resultados entre las dos entidades se debe a sus características heterogéneas, como se mencionó anteriormente. La EAS es una entidad que centra sus objetivos de acuerdo con los alcances contractuales, mientras que la FSI es una organización de carácter privado que ofrece servicios integrales enfocados al bienestar campesino. Se requieren procesos minuciosos de concientización para que los funcionarios de las entidades sean analíticos y autocríticos para aportar al mejoramiento continuo.

El diseño del modelo MECATA tiene como base la correlación identificada entre los agentes ejecutores y los resultados obtenidos del servicio en estudios que evalúan la calidad de servicios educativos, deportivos y sanitarios con el modelo EFOM (Zardoya-Alegría et al., 2004; Zardoya-Alegría et al., 2007; Delgado et al., 2007; Martínez-Moreno et al., 2012).

El proceso de evaluación mediante el Modelo MECATA se fundamenta en variables cualitativas y cuantitativas obtenidas a partir de interacciones con los usuarios, asistentes técnicos y la entidad prestadora de SATA.

Las instituciones y expertos coinciden con los extensionistas en que existe la necesidad de reconocer e incentivar el desempeño y obtención de logros, la forma de difusión del conocimiento con metodologías 


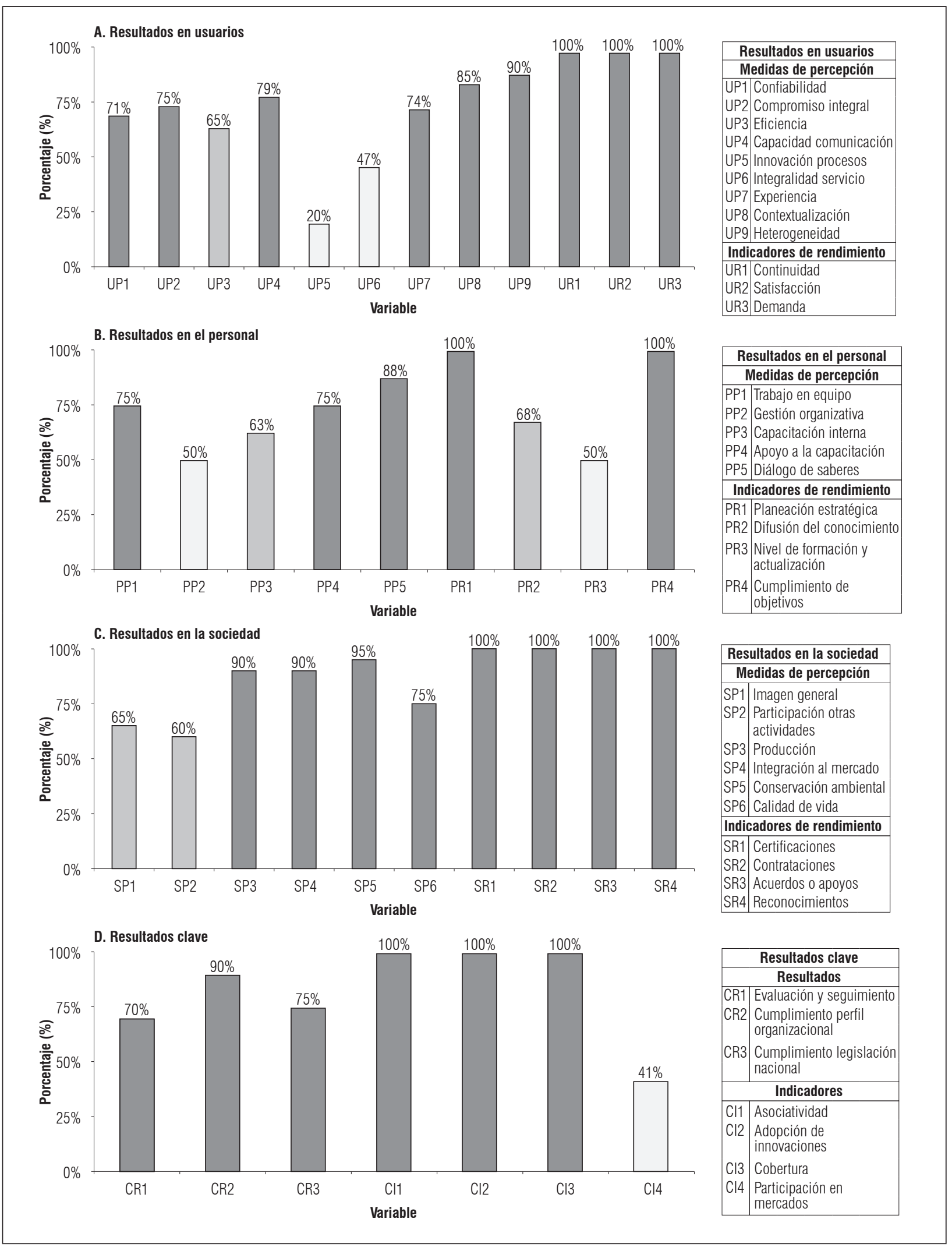

Figura 8. Resultados de evaluación de la calidad de SATA. FSI. 
participativas e individuales apropiadas. Así como, fomentar y fortalecer la asociatividad de los productores (Landini, 2012).

\section{CONCLUSIONES}

El modelo MECATA se ajusta a las necesidades de diversas entidades prestadoras de ATA por ser dinámico y flexible, estimular la reflexión, la participación y el debate en torno a un análisis objetivo, autocrítico y sensato, a partir de perspectivas internas y externas a la entidad.

El modelo es una herramienta que confiere un gran valor agregado, debido a que involucró procesos participativos como el Foro Nacional Linkata, además de, técnicas participativas comunitarias, donde se identificaron las necesidades y realidades de los actores que hacen parte del proceso de prestación del SATA en su contexto.

La captura de la percepción de los usuarios y asistentes técnicos, junto con la revisión documental, complementan el componente de evaluación cuantitativo del modelo y permiten determinar el grado de satisfacción de las necesidades de los usuarios del SATA, de sus familias y de la sociedad.

El puntaje final obtenido por las entidades puede considerarse como parámetro inicial para efectuar seguimiento a la calidad del desempeño del SATA puesto que la aplicación del modelo identifica fortalezas y debilidades, además del planteamiento de acciones de mejoramiento en diferentes ámbitos y proyección de impactos a mediano plazo.

\section{AGRADECIMIENTOS}

Los autores agradecen el financiamiento parcial otorgado con fondos institucionales de la Universidad $\mathrm{Na}$ cional de Colombia y la disponibilidad de los usuarios del servicio, asistentes técnicos y directivos de la Epsagro Agroéxito Sugamuxi en Pauna y de la Fundación San Isidro en Duitama del departamento de Boyacá.

Conflicto de intereses: el manuscrito fue preparado y revisado con la participación de los autores, quienes declaran no tener algún conflicto de interés que coloquen en riesgo la validez de los resultados aquí presentados.

\section{REFERENCIAS BIBLIOGRÁFICAS}

Álvarez R., A. 1989. La técnica nominal de grupo participativo. Seminario Taller: El Plan Nacional de Rehabilitación, PNR y la participación del IICA. Instituto Interamericano de Cooperación para la Agricultura, IICA, Medellín, Colombia

Cabo Salvador, J., J. Herreros G., V. Cabo M., M.A. Bellmont L., E. García J. y M. Vilches M. 2014. Plan estratégico de gestión de las organizaciones Sanitarias. Ediciones Díaz de Santos, Madrid, España.

Castaño-Reyes, G.P. 2016. Calidad del servicio de asistencia técnica agropecuaria: Análisis de dos casos en Boyacá. Tesis de maestría. Facultad de Ciencias Agrarias, Universidad Nacional de Colombia, Bogotá, Colombia.

Corpoica (Corporación Colombiana de Investigación Agropecuaria). 2015. Ciencia, tecnología e innovación en el sector agropecuario, diagnóstico para la misión para la transformación del campo. Corpoica, Bogotá, Colombia.

Cuevas Reyes, V., J. Baca del Moral, F. Cervantes Escoto y J. Aguilar Ávila. 2012. Asistencia técnica en el sector agropecuario en México: Análisis del VIII censo agropecuario y forestal. Rev. Mex. Cienc. Agric. 3(5), 943-957.

Delgado, M., E. Ramos y A. Pretel. 2007. Nuevas tendencias en evaluación de programas de desarrollo rural territorial: un proceso de autoevaluación en el Altiplano de Granada. Rev. Esp. Estud. Agrosoc. Pesqueros 213,11-40.

Gobernación de Antioquia. 2014. Informe de seguimiento y evaluación al Servicio de Asistencia Técnica Directa Rural, año 2013, en el departamento de Antioquia. Secretaria de Agricultura y Desarrollo Rural de Antioquia; Universidad de Antioquia, Medellín, Colombia.

Gutiérrez-Vanegas, B., L. Álvarez R., R. Pérez P., I. Parra C. y S. Cristancho M. 2013. Perfil y normas de competencia laboral para profesionales asistentes técnicos agropecuarios. Corpoica, Bogotá.

Jiménez G., Y.I., M.A. González R. y J. Hernández. 2016. De frente a la reforma educativa de segunda generación para la educación superior: żse han logrado los cambios en la epistemología docente? Perfiles Educ. 38(154), 20-40.

Landini, F. 2012. Problemas en la extensión rural paraguaya: modelos de extensión en la encrucijada. Cuad. Desarro. Rural 9(69), 127-149.

Lima de Almeida, A.C. y A.J. Gaia Cardoso. 2014. Diagnóstico rápido participativo e Matriz SWOT: estratégias de planejamento estratégico com base na atual posição do curso de Secretariado executivo UEPA. Rev. Gestão Secretariado 5(2), 117-137. Doi: 10.7769/gesec. v5i2.283 
Martínez-Moreno, A., V. Morales, C. Sánchez-Roca y F. Sánchez-Pérez. 2012. El modelo EFOM, análisis de los criterios resultados en un servicio deportivo municipal con más de 25.000 habitantes. Cuad. Psicol. Deporte 12(2), 71-76.

Méndez Sastoque, M.J. 2015. Satisfacción con el empleo de extensionista rural: un estudio cualitativo en Caldas, Colombia. Rev Ceres. 62(3), 241-250. Doi: 10.1590/0034-737X201562030003

Miles, M., M. Huberman y J. Saldaña. 2013. Qualitative data analysis: a methods sourcebook. $3^{\text {rd }}$ ed. Sage Publications, Thousand Oaks, USA.

MADR (Ministerio de Agricultura y Desarrollo Rural). 2002. Decreto 3199 de 2002, Decreto reglamentario de la prestación del Servicio Público Obligatorio de Asistencia Técnica Directa Rural previsto en la Ley 607 de 2000. Bogotá, Colombia.

MADR (Ministerio de Agricultura y Desarrollo Rural). 2005. Resolución 189 de 2005. Procedimiento y requisitos para la acreditación de las entidades prestadoras del servicio de asistencia técnica. Bogotá, Colombia.

Panayides, P. 2013. Coefficient alpha: Interpret with caution. Eur. J. Psychol. 9(4), 687-696. Doi: 10.5964/ejop. v9i4.653
RELASER (Red Latinoamericana para Servicios de Extensión Rural). 2012. Notas de Políticas, Institucionalidad de la Extensión Rural y las relaciones público-privadas. Relaser-Rimisp, Santiago, Chile. Doi: 10.5964/ejop. v9i4.653

Terrones-Cordero, A. 2013. Planeación participativa para elaborar un plan de desarrollo municipal: el caso de Acaxochitlán. Hidalgo. Econ. Soc. Territ. 13(42), 521559. Doi: 10.22136/est00201358

Vargas del Valle, R., S. Perry-Rubio, A. Cardona-Arango, D. Escobar-Uribe y U. Ramírez. 1996. Evaluación de las Unidades Municipales de Asistencia Técnica Agropecuaria UMATA. Vol. 2. Factores de éxito y fracaso de las UMATA. PBEST Asesores Ltda., Bogotá, Colombia.

Zardoya-Alegría, A., I.R. Guevara-Graterón, J. García-Bernal y M. Marzo-Navarro. 2004. Gestión de la calidad de los servicios sanitarios: Caso de autoevaluación según el EFOM. Rev. Iberoam. Contabilidad Gestión $2(4), 13-40$

Zardoya-Alegría, A., I.R. Guevara-Graterón, J. García-Bernal y M. Marzo-Navarro. 2007. El modelo EFOM en el sector salud: análisis de los criterios facilitadores. Cuad. Cienc. Econ. Empres. 52, 103-125. 